\title{
GIS-based Valuation of Ecosystem Services in Mountain Regions: A Case Study of the Chepelare Municipality in Bulgaria
}

\author{
Ivanova $\mathrm{E}^{1}$., Koulov B ${ }^{2}$, Borisova B. ${ }^{3}$, Assenov A. ${ }^{3}$, Vassilev K. ${ }^{4}$
}

\begin{abstract}
This study imports the Ecosystem Services concept in the economic valuation of the resources of a typical mountain municipality in Bulgaria. It applies a GIS-based approach and employs a system of methods, which include benefit transfer, market price, and contingent valuation, as well as local survey and statistical data for the following key ecosystem services: timber production, forest/agricultural products, and tourism and recreation. The investigation interprets the CORINE Land Cover (2012) classes as spatial units of identification, analysis, and valuation of selected Ecosystem Services, on which the welfare of local mountain population depends. Its results, among which is a map of the total economic value of a set of ecosystem services in the Municipality of Chepelare, is intended to support sustainable local governance in mountain regions.
\end{abstract}

Key words: ecosystem services valuation, sustainable governance, GIS, mountain regions, Bulgaria.

\section{Introduction}

The concept of ecosystem services (ES) is a topical idea that is quick to focus research attention on the development of an interdisciplinary, integrated methodological basis for geospatial research in support of sustainable development. It introduces a new perspective in the analysis of interactions between economic and natural systems with an emphasis on the sustainability of natural assets use and increase of its importance in strategic decision making. Anthropocentric analysis of natural assets in view of the diversity of their features, conditions, and resources and interpreting them as public "services" are among the notable features of the concept. ES have a high indicative potential, as the goal is oriented towards managing communication of past, present and future states of the nature- anthropogenic systems (Muller \& Burkhard, 2012). According to Honey-Rosés \& Pendleton (2013), ES research should provoke the interest of decision makers, including suggested action timeframes.

ES are defined as contributions of ecosystem structures and functions, in combination with other elements (e.g., anthropogenic - author's note), to human well-being (Burkhard et al., 2014). Ongoing development of the concept stimulates scientific debate about

| ${ }^{1}$ Space Research and Technology Institute, Bulgarian Academy of Sciences, Sofia, Bulgaria

${ }^{2}$ National Institute of Geophysics, Geodesy and Geography, Bulgarian Academy of Sciences, Sofia, Bulgaria

${ }^{3}$ Faculty of Geology and Geography, Sofia University "St. Kliment Ohridski”, Sofia, Bulgaria

${ }^{4}$ Institute of Biodiversity and Ecosystem Research, Bulgarian Academy of Sciences, Sofia, Bulgaria. 
convergence, even unification of priorities of the natural and social sciences and intensification of their interaction in order to achieve sustainable environment - an environment where ecological integrity and basic human needs are both simultaneously and consistently maintained for generations (Forman, 1995).

This study supports governance of local development in mountain conditions. Mountains are an important source of natural capital whose economic significance far exceeds their territorial scope. Typical for these areas is a higher dependence of the local population on the available ES and their quality management, which raises the salience of realistic economic valuation. The process of valuation itself depends on three main factors: 1. Unequivocal determination of the nature of the available ES and their reduction to economic products and services; 2. Physical definition of the "production boundary", according to the meaning of the Common International Classification of Ecosystem Services (CICES) and the Mapping and Assessment of Ecosystem Services (MAES 2013), within the ecosystems that generate the respective services; 3. Identification of the spatial distribution of the beneficiaries and ascertainment of their access to ES. Other challenges are the selection of the scale of research and geospatial units of analysis and evaluation.

This study aims at economic valuation of a complex of ES within a typical mountain municipality. The research focus is placed on their supply and spatial distribution. It adheres to the CICES classification and uses a local scale of research, which reflects on the selected indicators and parameters for evaluation. ES were evaluated both in biophysical and economic units. Expansion of the knowledge base to support municipal policy planning and governance of the territory is among the expected research results. The main tasks include: a. Identification of ES, which are crucial to the welfare of the local population and serve as basis for the local economy; b. Selection of informative biophysical assessment indicators that are supported by economic indicators which are traditionally monitored by Bulgarian statistics; c. Calculation, mapping and spatial analysis of the total supply and economic value of the ES complex within the mountain municipality.

\section{Study area}

The Chepelare Municipality covers an area of approximately $375 \mathrm{~km}^{2}$ and is located in the middle of the Rhodope Mountain in South Bulgaria. The municipality had 4794 inhabitants, $70 \%$ of which live in the municipal center of Chepelare (NSI 2013). The number of residents strongly vary during the year, due to the relatively high number of visitors, mainly tourists.

The municipality natural resource potential is characteristic for the Western Rhodope region, as well as for the Balkan mountain regions of similar altitude. This fact determines the strong dependence of the local economy on the natural ecosystems. The relief is quite diverse and its vertical segmentation is significant, as altitude within the municipality varies from $630 \mathrm{~m}$ in the Chepelarska River Valley to $2100 \mathrm{~m}$ at the mountain ridge. The local climate exhibits mountain characteristics, but is still relatively mild, with a larger number of sunny days per year, due to the significant influence of the Aegean Sea. 
A significant majority of the municipality's territory $(70 \%)$ is covered by natural forest vegetation, about $74 \%$ of which - coniferous species (Pinus sylvestris, Picea abies, Pinus peuce, Pinus heldreichii). Deciduous forests, which occupy less than $9 \%$ of the forest area, are represented by the species Fagus sylvatica, Carpinus betulus and Betula pendula. In the deciduous vegetation belt arrays of black pine (Pinus nigra) are artificially planted. A wide variety of species of herbaceous and shrub stock characterize the area, many of which have medicinal properties and are on restricted gathering regime.

The ecological diversity of the municipality, determined by its mountainous topography, climate and wild life, is an excellent prerequisite for the development of tourism, recreation and various sports activities. Tourism is one of the main economic sectors: a significant part of the Pamporovo Resort - one of the three largest mountain resorts of Bulgaria - is located within the studied municipality, while the municipal center of Chepelare has also developed as an international ski destination. About $30 \%$ of the tourists come from abroad (Municipality Development Plan [MDP] 2014-2020). In addition to the various mountain sports, alternative types of tourism - eco, rural, and cultural - are increasingly practiced. The tourism and recreation capacities of the municipality are unevenly used - mainly around the two main tourist destinations - the Pamporovo Tourist Resort and the Town of Chepelare. In the last few years village tourism, as well as second homes in other villages, feature quite successfully, aided by development of broadband internet and the Chepelare Tourist Information Center.

The other sectors of the municipal economy are also closely related to the provision and use of local ES. Forestry is the second most important economic branch, in which at least six forest owners' cooperatives are engaged. Mainly logging, wood processing, and ski manufacturing, and to a lesser extent gathering of forest food products and hunting, are typical areas of employment for the local population. Subsistence agriculture is typical for the municipality. The ES in the Chepelare Municipality provide an excellent forage base for raising of livestock, but the specific weather conditions in the mountains hinder crop growing. Potatoes are the main product, grown for household purposes. Commercial agriculture and the related food industry are almost nonexistent, mainly due to the unfavorable market conditions.

\section{Data and methods}

\subsection{Data and sources}

The study relies on a rich information basis, which includes results from a biophysical assessment of ES from several landscape-environmental and socio- and economic geographical investigations of the case study area. Primary importance is given to the gradual expansion of the analytic base with information about practical problems in the management of natural assets, shared by the local business community. These become the basis for selection of economic analysis and evaluation methods and conducting series of surveys and interviews.

The economic assessment of ES requires a wide range of data for particular timeframes and geographic scales of aggregation. The investigation encountered serious difficulties in obtaining and gathering such statistical data at the local level, due mostly to legal restrictions to its access and use. The available data at regional, national, and local level 
was supplemented with data from field studies and surveys of the local population, government and business circles. The resulting information base acted as a limiting factor in the selection of ES for evaluation (Table 1).

The study focuses on five classes (CICES; MAES, 2013) of representative Provisioning and Cultural ES (timber, wood fuel, freshwater, food (livestock, crops, and natural plants (herbs), recreation and tourism. The economic data used is referenced, according to eight biophysical indicators (adapted as per Burkhard et al., 2014) (Table 2). The team conducted a field study targeted on genetic resourceson the ground of existing biotopes, habitat diversity and landscape biodiversity, which is to be published separately. The data deficit did not allow the valuation of fodder - an important ES, especially for mountain regions. This is yet another reason for advocating a continuous monitoring of mountain regions.

The lack of appropriate data about the Supporting ES postpones their valuation to a subsequent stage of the study, which will include modeling in a GIS environment.

Table 1. Ecosystem Services, Representative of the Chepelare Municipality

\begin{tabular}{|c|c|c|c|c|}
\hline \multirow{3}{*}{$\begin{array}{l}\text { CLC } \\
2012 \\
\text { code }\end{array}$} & \multirow{3}{*}{$\begin{array}{l}\text { Area } \\
\text { (ha) }\end{array}$} & \multicolumn{3}{|c|}{$\begin{array}{l}\text { Ecosystem Services, Representative of the Chepelare Municipality, generated by } \\
\text { the landscapes in the respective land cover class (CLC) }\end{array}$} \\
\hline & & \multirow{2}{*}{$\begin{array}{l}\text { Potentially Delivered Ecosystem } \\
\text { Services }\end{array}$} & \multicolumn{2}{|c|}{ Actually Delivered Ecosystem Services } \\
\hline & & & $\begin{array}{l}\text { Statistical data is } \\
\text { available }\end{array}$ & $\begin{array}{l}\text { Statistical data is } \\
\text { not available }\end{array}$ \\
\hline 312 & 19971 & \multirow{3}{*}{$\begin{array}{l}\text { Timber and Wood fuel; Game, Berries } \\
\text { and Mushrooms; Genetic materials } \\
\text { from all biota; Freshwater; Local } \\
\text { climate regulation; Water flow } \\
\text { regulation; Air quality regulation; Water } \\
\text { purification; Erosion regulation; } \\
\text { Natural hazard protection; Landscape } \\
\text { aesthetic, amenity and inspiration; } \\
\text { Recreation and tourism; Natural } \\
\text { heritage and natural diversity }\end{array}$} & $\begin{array}{l}\text { Timber; Freshwater; } \\
\text { Mushrooms }\end{array}$ & \multirow{3}{*}{$\begin{array}{l}\text { Game; Mass flow } \\
\text { regulation; Water } \\
\text { purification; Natural } \\
\text { hazard protection; } \\
\text { Landscape aesthetic, } \\
\text { amenity } \\
\text { inspiration and }\end{array}$} \\
\hline 311 & 2352 & & $\begin{array}{l}\text { Timber; Freshwater; } \\
\text { Mushrooms }\end{array}$ & \\
\hline 313 & 4668 & & $\begin{array}{l}\text { Wood fuel; } \\
\text { Freshwater; } \\
\text { Mushrooms; Berries }\end{array}$ & \\
\hline 321 & 1741 & $\begin{array}{l}\text { Fodder; Natural plants (herbs) used in } \\
\text { medicine and/or cosmetics; Genetic } \\
\text { materials from all biota; Freshwater; } \\
\text { Local climate regulation; Water flow } \\
\text { regulation; Water purification; } \\
\text { Pollination; Natural heritage and } \\
\text { natural diversity }\end{array}$ & $\begin{array}{l}\text { Livestock (domestic); } \\
\text { Freshwater; Natural } \\
\text { plants (herbs) }\end{array}$ & $\begin{array}{l}\text { Natural plants } \\
\text { (herbs) used in } \\
\text { medicine and/or } \\
\text { cosmetics; Fodder; } \\
\text { Pollination; Species } \\
\text { diversity; Landscape } \\
\text { aesthetic, amenity } \\
\text { and inspiration }\end{array}$ \\
\hline 231 & 93 & $\begin{array}{l}\text { Fodder; Natural plants (herbs) used in } \\
\text { medicine and/or cosmetics; Pollination; } \\
\text { Landscape aesthetic, amenity and } \\
\text { inspiration; Natural heritage and natural } \\
\text { diversity }\end{array}$ & $\begin{array}{l}\text { Livestock (domestic); } \\
\text { Freshwater }\end{array}$ & $\begin{array}{l}\text { Natural plants } \\
\text { (herbs) used in } \\
\text { medicine and/or } \\
\text { cosmetics; } \\
\text { Fodder; Pollination; } \\
\text { Species diversity }\end{array}$ \\
\hline 242 & & & ater & \\
\hline 243 & 3454 & & Crops; Freshwater & \\
\hline 112 & 431 & $\begin{array}{l}\text { Recreation and tourism; Cultural } \\
\text { heritage; }\end{array}$ & $\begin{array}{l}\text { Recreation and } \\
\text { tourism; Freshwater }\end{array}$ & Cultural heritage \\
\hline 142 & 132 & Recreation; Air quality regulation; & $\begin{array}{l}\text { Recreation and } \\
\text { tourism; Freshwater }\end{array}$ & Cultural heritage \\
\hline
\end{tabular}




\begin{tabular}{|l|l|l|l|l|}
\hline 324 & 5317 & $\begin{array}{l}\text { Natural plants (herbs) used in medicine } \\
\text { and/or cosmetics; Berries and } \\
\text { mushrooms; Water flow regulation; } \\
\text { Erosion regulation }\end{array}$ & $\begin{array}{l}\text { Livestock (domestic); } \\
\text { Natural plants } \\
\text { (herbs); Freshwater }\end{array}$ & $\begin{array}{l}\text { Natural plants } \\
\text { (herbs) used in } \\
\text { medicine and/or } \\
\text { cosmetics; } \\
\text { Pollination }\end{array}$ \\
\hline 333 & 34 & $\begin{array}{l}\text { Natural plants (herbs) used in medicine } \\
\text { and/or cosmetics }\end{array}$ & $\begin{array}{l}\text { Livestock (domestic); } \\
\text { Natural plants } \\
\text { (herbs); Freshwater }\end{array}$ & $\begin{array}{l}\text { Natural plants } \\
\text { (herbs) used in } \\
\text { medicine and/or } \\
\text { cosmetics; } \\
\text { Pollination }\end{array}$ \\
\hline
\end{tabular}

\subsubsection{Indicators for Assessment of Provisioning Ecosystem Services}

Industrial logging, as well as gathering of firewood for household use feature among the Provisioning ES of greatest importance for the Municipality of Chepelare. National Statistical Institute (NSI 2015b) data for 2012 is used in the assessment of this industry's productivity (Table 3). Field studies, MDP 2014-2020, and Hvoina State Forestry Farm (2014) data enabled the evaluation of the household firewood capacity.

Table 2. ESs classification (adapted to CICES v4.3 (MAES, 2013) and Burkhard et al., 2014) and valuation technics

\begin{tabular}{|c|c|c|c|c|c|c|c|}
\hline \multicolumn{4}{|c|}{ Ecosystem services } & \multirow[b]{2}{*}{$\begin{array}{l}\text { Biophysical } \\
\text { indicators }\end{array}$} & \multirow[b]{2}{*}{ Unit } & \multirow[b]{2}{*}{ Economic indicator } & \multirow[b]{2}{*}{$\begin{array}{l}\text { Monetary } \\
\text { valuation } \\
\text { techniques }\end{array}$} \\
\hline 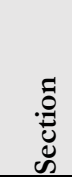 & $\frac{5}{0} \cdot \frac{0}{0}$ & 仓̊ & Class & & & & \\
\hline & & & & $\begin{array}{l}\text { Agricultural products } \\
\text { yield (potatoes) }\end{array}$ & $\mathrm{t} / \mathrm{ha} / \mathrm{yr}$ & $\begin{array}{l}\text { Market value of } \\
\text { agricultural products }\end{array}$ & Market price \\
\hline & & & $\begin{array}{l}\text { Food } \\
\text { Water }\end{array}$ & $\begin{array}{lr}\text { Domestic animals } \\
\text { products yield } \\
\text { milk, sheep milk, goat } \\
\text { milk, meat } & \text { from } \\
\text { cattle, meat } & \text { from } \\
\text { goats, greasy } & \text { wool, } \\
\text { honey, eggs) } & \end{array}$ & $\begin{array}{l}\text { l/ha/yr; } \\
\mathrm{kg} / \mathrm{ha} / \mathrm{yr} \text {; } \\
\text { number }\end{array}$ & $\begin{array}{l}\text { Economical income } \\
\text { per year; } \\
\text { Market value of } \\
\text { domestic animals } \\
\text { products milk }\end{array}$ & $\begin{array}{l}\text { Market price } \\
\text { Benefits } \\
\text { transfer } \\
\text { Contingent } \\
\text { valuation }\end{array}$ \\
\hline & & & & Mushrooms yield & $\mathrm{kg} / \mathrm{ha} / \mathrm{yr}$ & $\begin{array}{l}\text { Market value } \\
\text { mushrooms }\end{array}$ & \begin{tabular}{|l} 
Benefits \\
transfer \\
Contingent \\
valuation \\
\end{tabular} \\
\hline & & 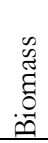 & & $\begin{array}{l}\text { Natural plants/herbs } \\
\text { yield (rosehip) }\end{array}$ & $\mathrm{kg} / \mathrm{ha} / \mathrm{yr}$ & $\begin{array}{l}\text { Market value of } \\
\text { rosehip }\end{array}$ & $\begin{array}{l}\text { Benefits } \\
\text { transfer } \\
\text { Contingent } \\
\text { valuation } \\
\end{array}$ \\
\hline 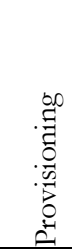 & : & 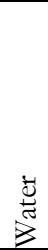 & $\begin{array}{l}\text { Groun } \\
\text { d water } \\
\text { for } \\
\text { drinkin } \\
g \\
\text { purpos } \\
\text { es }\end{array}$ & $\begin{array}{l}\text { Freshwater recharge } \\
\text { yield }\end{array}$ & $\mathrm{m}^{3} / \mathrm{ha} / \mathrm{yr}$ & $\begin{array}{l}\text { Market value of water } \\
\text { for domestic } \\
\text { consumption }\end{array}$ & Market price \\
\hline
\end{tabular}




\begin{tabular}{|c|c|c|c|c|c|c|c|}
\hline & \multirow[b]{2}{*}{ 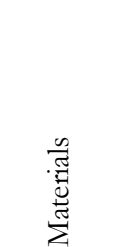 } & \multirow[b]{2}{*}{ 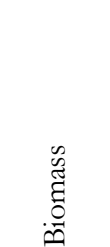 } & Timber & Yield of timber & $\mathrm{m}^{3} / \mathrm{ha} / \mathrm{yr}$ & \begin{tabular}{|lll}
$\begin{array}{l}\text { Market } \\
\text { timber }\end{array}$ & value & \\
\end{tabular} & Market price \\
\hline & & & $\begin{array}{l}\text { Firewo } \\
\text { od }\end{array}$ & Wood fuel yield & $\mathrm{m}^{3} / \mathrm{ha} / \mathrm{yr}$ & $\begin{array}{l}\text { Market value of water } \\
\text { for } \\
\text { purposes }\end{array}$ & $\begin{array}{l}\text { Market price } \\
\text { Benefits } \\
\text { transfer } \\
\text { Contingent } \\
\text { valuation }\end{array}$ \\
\hline 宓 & 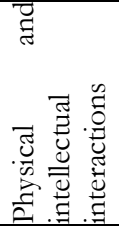 & 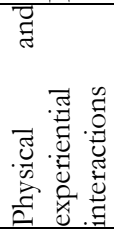 & $\begin{array}{l}\text { Recreat } \\
\text { ion and } \\
\text { tourism }\end{array}$ & $\begin{array}{l}\text { Number } \\
\text { accommodations }\end{array}$ & of $\begin{array}{ll}\text { Number of } \\
\text { accommod } \\
\text { ations } & \text { for } \\
\text { bed } & \text { per } \\
\text { year } & \end{array}$ & $\begin{array}{l}\text { Payment for tourism } \\
\text { services }\end{array}$ & $\begin{array}{l}\text { Market price } \\
\text { Contingent } \\
\text { valuation }\end{array}$ \\
\hline
\end{tabular}

The assessment of actual services provision in the Food class employs biophysical indicators, while the assessment of the Domestic Animal Products Yield indicator uses animal productivity 2013 data (MDP 2014-2020), relative to the size of their forage areas. Field research provided statistical data for the Mushroom Yield, Natural Plants Yield and Agricultural Products Yield indicators for 2009-2011 and 2013 respectively. The NSI (2015a) series on Prices of agricultural products serve as data source on the economic indicators.

One of the most important indicators for the development of the Chepelare Municipality is Groundwater for Drinking Purposes. Although there is a link between groundwater bodies and land cover (CIS for WFD, 2004), municipal drinking water supply cannot be directly tied to the CLC classes. These ES are calculated as the extracted water quantities for 2011 (MDP 2014-2020) are assigned to the operational resources of groundwater bodies (EABD 2010-2015) and the area they occupy within the municipality.

\subsubsection{Indicators for Assessment of Cultural Ecosystem Services}

The spatial distribution of the Cultural ES in the municipality relates to the Discontinuous Urban Fabric class, which incorporates all settlements, and the Sport and Leisure Facilities class, which, according to the CLC 2012 classification, includes the Pamporovo Tourist Resort, as well as the Town of Chepelare. Ample data is available about the tourist capacity of the municipality for 2013 (MDP 2014-2020) and the 2012 annual tourism revenues (NSI Regional Statistics).

\subsection{Methods of ecosystem services valuation}

This investigation works under the understanding that the valuation process is a regulatory adaptation which serves as a feedback mechanism in an economic system (TEEB, 2010). Using Barbier (2008) terminology, this economic assessment is specifically targeted to support the "sustainable" management of the ES flows in Chepelare Municipality and the sustainable governance of its environmental assets. The selection of assessment and valuation methods results from a preliminary analysis, which takes into account a number of factors (Brouwer et al., 2013). These are derived from the characteristics of the study area, among which availability of data and expertise, applicability of key indicators provided by the national statistical system, the possibility and practicality of transferring data or using generalizations, as well as the spatial 
variations of representative ES. On that basis, the study relies mostly on the method of market prices (Table 2). Despite its weaknesses, for example, its applicability to a limited number of ES, this method proves very informative and motivating in terms of decisionmaking at the local level.

For the purposes of this study emphasis is placed on the application of the contingent valuation method, which, despite the increase of subjectivity of the overall assessment, provides considerable information and adequately reflects the influence of natural heterogeneity on spatial disparities in the provision of ES and their interaction with the human factor. The methodology also includes the use of the transfer method, where data is used from municipalities with similar physical and human geographic characteristics from within the Rhodope Mountains.

Table 3. Valuation of the selected ecosystem services in Chepelare Municipality

\begin{tabular}{|c|c|c|c|c|c|c|c|c|c|c|}
\hline \begin{tabular}{|l} 
GIS \\
Lear
\end{tabular} & EC (i) & ES & BI & $\begin{array}{l}\text { Area } \\
\text { (ha) }\end{array}$ & BIV (s) & EIV (e) & $\begin{array}{l}\text { Income } \\
\text { (euro) }\end{array}$ & Year & $\begin{array}{l}\text { ESV } \\
\epsilon / \text { ha }\end{array}$ & $\begin{array}{l}\text { TEV }_{\mathrm{i}} \\
\epsilon / \mathrm{ha}\end{array}$ \\
\hline L1 & CLC 2012: 312 & \multirow[t]{2}{*}{ Timber } & $\begin{array}{l}\text { Coniferous } \\
\text { timber }\end{array}$ & 19971 & $\begin{array}{l}1,34^{1)} \\
\mathrm{m}^{3} / \mathrm{ha}\end{array}$ & $\begin{array}{l}43,972) \\
\boldsymbol{\epsilon} / \mathrm{m}^{3}\end{array}$ & 1176687,33 & $2012^{1)}$ & 58,91 & 58,91 \\
\hline $\mathrm{L} 2$ & CLC $2012-311$ & & \begin{tabular}{|l|} 
Deciduous \\
timber
\end{tabular} & 2352 & $\begin{array}{l}0,34^{1)} \\
\mathrm{m}^{3} / \mathrm{ha}\end{array}$ & $\begin{array}{l}34,51^{2)} \\
\boldsymbol{\epsilon} / \mathrm{m}^{3}\end{array}$ & 27596,96 & $2012^{1)}$ & 11,73 & 11,73 \\
\hline \multirow[t]{2}{*}{$\mathrm{L3}$} & \multirow[t]{2}{*}{$\begin{array}{l}\text { CLC 2012: 311, } \\
313\end{array}$} & \multirow[t]{2}{*}{$\begin{array}{l}\text { Firewood } \\
\text { Wood fuel }\end{array}$} & $\begin{array}{l}\text { Coniferous } \\
\text { firewood }\end{array}$ & \multirow[t]{2}{*}{7020} & $\begin{array}{l}0,13^{3)} \\
\mathrm{m}^{3} / \mathrm{ha}\end{array}$ & $\begin{array}{l}10,23^{2)} \\
\boldsymbol{\epsilon} / \mathrm{m}^{3}\end{array}$ & 9207 & \multirow[t]{2}{*}{$2012^{3)}$} & 1,31 & \multirow[t]{2}{*}{14,06} \\
\hline & & & \begin{tabular}{|l|} 
Deciduous \\
firewood
\end{tabular} & & $\begin{array}{l}0,71^{3)} \\
\mathrm{m}^{3} / \mathrm{ha}\end{array}$ & $\begin{array}{l}17,9^{2)} \\
\boldsymbol{\epsilon} / \mathrm{m}^{3}\end{array}$ & 89500 & & 12,75 & \\
\hline L4 & $\begin{array}{l}\text { CLC 2012: 311, } \\
312,313\end{array}$ & Mushrooms & Mushrooms & 26991 & $\begin{array}{l}0,11^{4)} \\
\mathrm{kg} / \mathrm{ha}\end{array}$ & $\begin{array}{l}5,11^{4)} \\
\boldsymbol{\epsilon} / \mathrm{kg}\end{array}$ & 14645,26 & $2009-$ & 0,54 & 0,54 \\
\hline \multirow[t]{6}{*}{ L5 } & \multirow[t]{6}{*}{$\begin{array}{l}\text { CLC 2012: } 231 \\
321\end{array}$} & \multirow{6}{*}{$\begin{array}{l}\text { Domestic } \\
\text { animals } \\
\text { products }\end{array}$} & Cow milk & \multirow[t]{6}{*}{1834} & $\begin{array}{l}571,43^{3)} \\
\text { l/ha }\end{array}$ & $0,30^{5)} \boldsymbol{\epsilon} / 1$ & 309331,55 & \multirow[t]{6}{*}{$2013^{3)}$} & 168,66 & \multirow[t]{6}{*}{298,75} \\
\hline & & & Sheep milk & & \begin{tabular}{|l|}
$65,433)$ \\
$1 / \mathrm{ha}$
\end{tabular} & $0,65^{5)} \boldsymbol{\epsilon} / 1$ & 78100,04 & & 42,58 & \\
\hline & & & $\begin{array}{|ll|}\text { Meat } & \text { from } \\
\text { cattle } & \\
\end{array}$ & & $\begin{array}{l}7,09^{3)} \\
\mathrm{kg} / \mathrm{ha}\end{array}$ & $\begin{array}{l}\left.2,39^{5}\right) \\
\boldsymbol{\epsilon} / \mathrm{kg}\end{array}$ & 31044,00 & & 16,93 & \\
\hline & & & $\begin{array}{|ll|}\begin{array}{l}\text { Meat } \\
\text { goats }\end{array} & \text { from } \\
\end{array}$ & & $\begin{array}{l}10,91^{3)} \\
\mathrm{kg} / \mathrm{ha}\end{array}$ & $\begin{array}{l}5,12^{5)} \\
\boldsymbol{\epsilon} / \mathrm{kg} \\
\end{array}$ & 102370,86 & & 55,82 & \\
\hline & & & Greasy wool & & \begin{tabular}{|l|}
$7,63^{3)}$ \\
$\mathrm{kg} / \mathrm{ha}$ \\
\end{tabular} & $\begin{array}{l}0,55^{5)} \\
\boldsymbol{\epsilon} / \mathrm{kg} \\
\end{array}$ & 7730,73 & & 4,22 & \\
\hline & & & Honey & & $\begin{array}{l}3,82^{3)} \\
\mathrm{kg} / \mathrm{ha}\end{array}$ & \begin{tabular}{|l|}
$2,76^{5}$ \\
$\boldsymbol{\epsilon} / \mathrm{kg}$ \\
\end{tabular} & 19321,21 & & 10,54 & \\
\hline \multirow[t]{2}{*}{ L6 } & \multirow[t]{2}{*}{ 2012: 242, } & $\begin{array}{l}\text { Agricultural } \\
\text { products }\end{array}$ & Potatoes & \multirow[t]{2}{*}{4066} & $\begin{array}{l}\left.75,22^{4}\right) \\
\mathrm{kg} / \mathrm{ha}\end{array}$ & $0,2^{5)} \boldsymbol{\epsilon} / \mathrm{kg}$ & 60990 & $2013^{4)}$ & 15 & \multirow[t]{2}{*}{18,5} \\
\hline & & $\begin{array}{l}\text { Domestic } \\
\text { animals } \\
\text { products }\end{array}$ & Eggs & & $\begin{array}{l}54,6^{3)} \\
\mathrm{n} / \mathrm{ha}\end{array}$ & $0,06^{5)} \boldsymbol{\epsilon} / \mathrm{n}$ & 14248,51 & $2013^{3)}$ & 3,50 & \\
\hline \multirow[t]{2}{*}{ L7 } & \multirow[t]{2}{*}{ 2012: 324} & $\begin{array}{l}\text { Domestic } \\
\text { animals } \\
\text { products }\end{array}$ & Goat milk & 5351 & $\begin{array}{l}2,24^{3)} \\
1 / \mathrm{ha}\end{array}$ & $0,31^{5)} \boldsymbol{\epsilon} / 1$ & 3731,00 & $2013^{3)}$ & 0,70 & \multirow[t]{2}{*}{0,76} \\
\hline & & \begin{tabular}{|l|} 
Natural \\
plants \\
(herbs)
\end{tabular} & Rosehip & & $\begin{array}{l}0,21^{4)} \\
\mathrm{kg} / \mathrm{ha}\end{array}$ & $\begin{array}{l}0,31^{4)} \\
\boldsymbol{\epsilon} / \mathrm{kg}\end{array}$ & 321 & $\begin{array}{l}2009-1 \\
2011^{4)}\end{array}$ & 0,06 & \\
\hline \multirow[t]{4}{*}{ L8 } & \begin{tabular}{|l|} 
Bogutevo \\
CLC2012: 112 \\
\end{tabular} & \multirow[t]{4}{*}{$\begin{array}{l}\text { Recreation } \\
\text { and tourism }\end{array}$} & \multirow{4}{*}{$\begin{array}{l}\text { Number } \\
\text { accommo- } \\
\text { dations }\end{array}$} & $\mathrm{f} 30$ & 33) beds & \multirow{4}{*}{$\begin{array}{l}\left.1030,28^{6}\right) \\
\epsilon \text { for bed } \\
\text { per year }\end{array}$} & 3090,84 & \multirow[t]{4}{*}{$\begin{array}{l}2012^{6)} \\
2013^{3)}\end{array}$} & 103,03 & 103,03 \\
\hline & \begin{tabular}{|l} 
Zabardo, \\
CLC2012: 112
\end{tabular} & & & 33 & 203) beds & & 20605,60 & & 624,41 & 624,41 \\
\hline & \begin{tabular}{|l} 
Zornitzha \\
CLC2012: 112
\end{tabular} & & & 30 & 143) beds & & 14423,92 & & 480,80 & 480,80 \\
\hline & \begin{tabular}{|l} 
Orehovo \\
CLC2012: 112
\end{tabular} & & & 34 & $\begin{array}{l}101^{3)} \\
\text { beds }\end{array}$ & & 104058,28 & & 3060,54 & 3060,54 \\
\hline
\end{tabular}




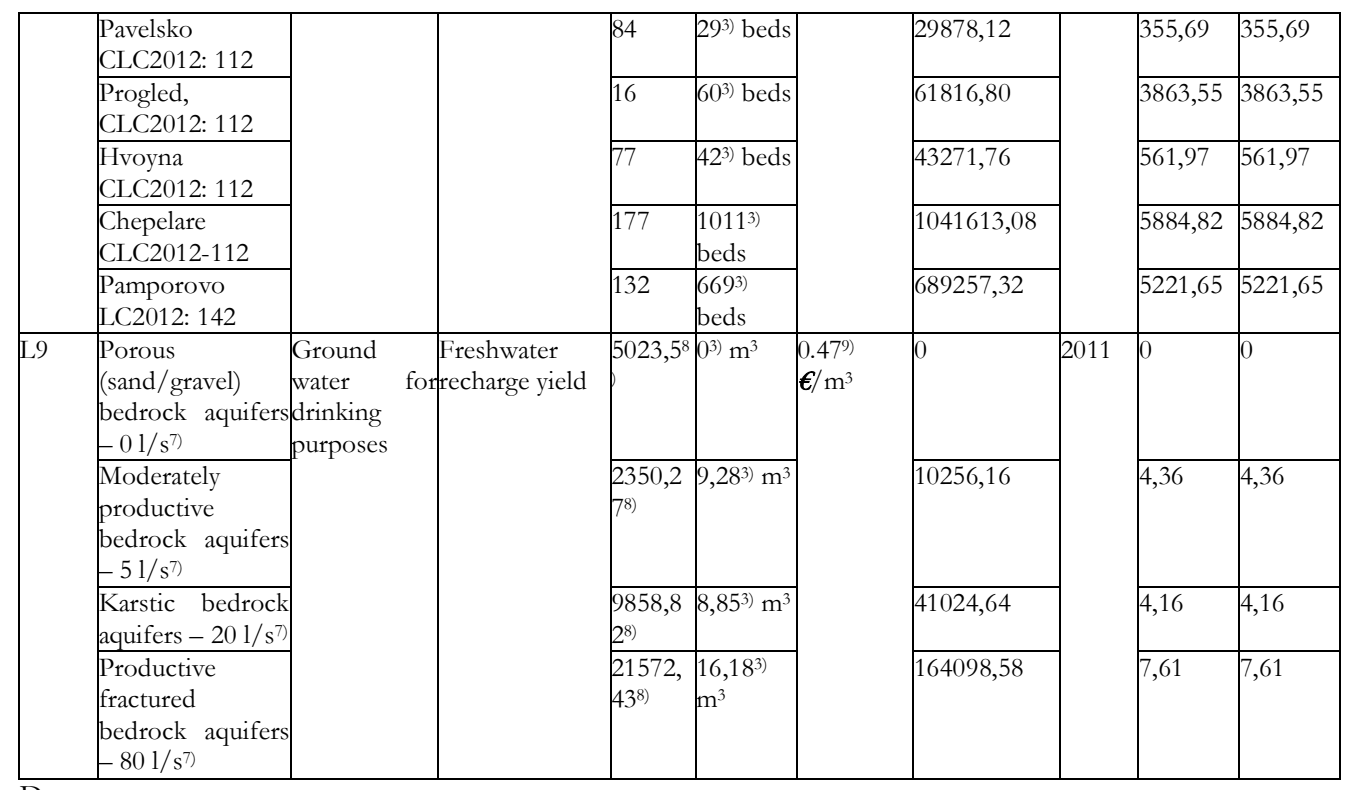

Data sources:

1) National Statistical Book, NSI, 2015, Sofia; 2) Price list of Hvoyna State Foresty, 2014, Chepelare; 3) Municipal Development Plan of the Chepelare Municipality 2014-2020, BG051PO002/ 13/1.3-07, 2015, Chepelare; 4) Local surveys and interviews; 5) National Statistical Institute, Republic of Bulgaria: Prices of agricultural production 2015 (data series); 6) National Statistical Institute, Republic of Bulgaria: Regional statistics (data series); 7 Management plane for the river basins in the East Aegean Basin Directorate 2010-2015, Ministry of Environment and Waters, Bulgaria; 8) Geological map of Bulgaria, scale 1:50 000; 9) State Commission for Energy and Water Regulation, Bulgaria, Resolution № 043/30.07.2008

\subsection{Geo-database and approach application in GIS}

The study uses a GIS-data set representing the land cover of Bulgaria for the year 2012 for spatial analysis and mapping of ecosystem services in the Municipality of Chepelare (National Reference Centre, Executive Environmental Agency at the Bulgarian Ministry of Environment and Waters). The polygons are divided among 35 classes of the CORINE Land Cover classification which are present in Bulgaria. The minimum map unit is $25 \mathrm{ha}$. The conceptual scale is 1:100 000. The survey area includes eleven CLC 2012 classes from level 3 (Table 1). Groundwater bodies are mapped on the basis of the geological map of Bulgaria, M1: 50000 (Kozhuharov et al., 1989, 1991). CIS WFD 2000/60 / EC delineation methodology is used for identification of the four groundwater bodies in the researched area, which are evaluated in terms of provision of fresh water for drinking purposes.

The methodological decisions are directly influenced by the possibilities of mapping ES on the basis of an integrated biophysical and economic evaluation (Hayha, 2014). As a first step, data is organized in a common geo-database in UTM WGS-84 Zone 35T coordinate system. The information on the biophysical and economic indicators is transferred to the attributive tables of the respective GIS layers. The value of the ES for each indicator is calculated as: 


$$
E S V=s * e
$$

where: ESV is Ecosystem service value; $s$ is biophysical indicator value; $e$ is economic indicator value, and

$$
T E V_{i}=\sum_{j=0}^{m} E S V_{j}
$$

where: TEV $V_{i}$ is total economic value of ecosystem class $i$; ESV $j$ is ES value; and $m$ is the number of biophysical indicators in ecosystem class $i$.

The study generated nine raster layers $\left(L_{i}\right)$ with pixel size 20x20 meters which contain information on valuated ecosystem services in $€ / \mathrm{ha} / \mathrm{yr}$. For each class of ecosystems (Table 3). The final economic value (TEV) of ecosystem services in $€ / \mathrm{ha} / \mathrm{yr}$ for the Chepelare Municipality ecosystems is calculated as:

$$
T E V=\sum_{i=0}^{n} L_{i} \quad\left\{\begin{array}{l}
L_{i}=T E V_{i} \text { for valuated ecosystems } \\
L_{i}=0 \text { for non valuated ecosystems }
\end{array}\right.
$$

where: TEV is total economic value of ecosystem services; $L_{i}$ is the pixel value of the GIS-layer $i$; and $n=9$ is the number of GIS-layers.

The application of the described model generates a map of the total economic value of a set of ecosystem services in the Municipality of Chepelare (Figure 1). 

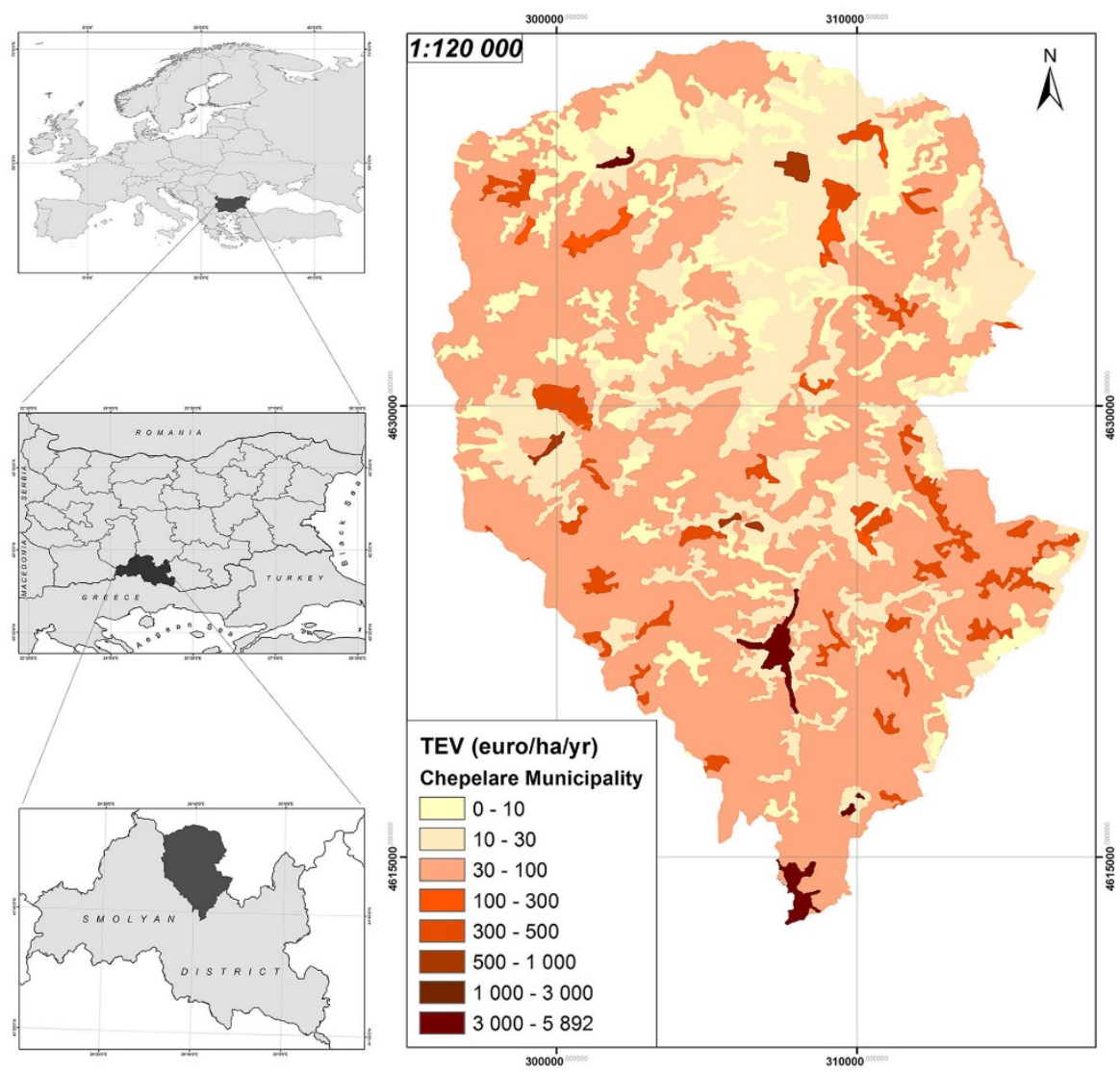

Figure 1. Total Economic V alue (TEV) of the selected ecosystem services in Chepelare Municipality

\section{Conclusions}

The selected basic ES account for an annual revenue of $€ 4.2$ million in the municipal economy. The largest contributions come from the following three services: Recreation and Tourism, Logging, and Domestic Animals Products (Table. 3). It is hardly a coincidence that nearly $67 \%$ of the villages of the municipality plus the Pamporovo Resort and the Town of Chepelare are actively engaged in providing tourist services (Table. 3). The value of the Recreation and Tourism class of ES, which produces an annual income of $€ 2$ million, provides ample evidence of the large potential of the Cultural Services in mountain conditions. Logging and Domestic Animals services secure an income of, respectively, about $€ 1.2$ million and $€ 878$ thousand per year.

In spatial perspective, the total economic value (TEV) per year of the selected ES in the Municipality of Chepelare varies from $€ 0.76$ to $€ 5,892$ per hectare (Figure 1). Naturally, the highest TEV is characteristic for the Urban Systems class. This high territorial concentration of economic value is due to reasons that vary from the small size of this most anthropogenized ecosystem class to the current methods of accounting and the fact that settlements "absorb" all financial flows of the surrounding areas. Based on the 
touristic capacity of this ecosystem class, the value varies from about $€ 100$ to approximately $€ 5900$ per hectare annually.

The TEV of the Domestic Animals Products services, provided by the ecosystems within the classes of Natural Grassland and Pastures (CLC 2012), is approximately $€ 300$ / ha / year. Due largely to the difficulties of valuation of all forest services, as well as to the low market price of wood, the TEV of forest ecosystems is quite low - from about $€ 15$ to about $€ 60 /$ ha / yr. It is worth noting that the upcoming more comprehensive evaluation of the supporting ecosystem services is expected to raise dramatically the TEV of these particular ecosystems. In such a case, a rethinking of the economic strategy of both the Chepelare Municipality, as well as the territorial units of higher territorial level is unavoidable, leading to a more sustainable governance of the forestry, waters, and the energy sector. Especially promising is the production of clean and renewable energy, as well as the promotion of innovative higher-yielding alternatives associated with the genetic resources of mountain municipalities.

\section{Discussion and conclusion}

The results point to the following limitations of economic ES valuation: a. Methodological problems which stem from the current ES classification, e.g., the reduction of services to "products" - a rather questionable concept from an economic point of view; b. Data shortages in respect to basic services, such as Foods, Fodder, Pollination, Species Diversity, Natural Plants (herbs) used in medicine and / or cosmetics. The latter are due to EU statistical regulations and are most pronounced at the local level. The above limitations do not allow for the time being the valuation to include a number of ES, mainly produced by the regulation and maintenance, and the cultural and aesthetic ecosystem functions, which are currently "invisible" for the market. Nevertheless, the proposed GIS-aided model of spatial assessment of ES not only will assist local authorities in planning future municipal development, but also serve as a reliable benchmark for future valuation research, especially in bringing to light a substantial share of currently undervalued ES.

Despite the limitations, economic valuation has the potential to become an even more powerful factor, which will stimulate public interest in the concept of ES and support sustainable governance of local development. This study is primarily based on a selection of ES that are clearly marketable and for which data is publicly accessible. Preliminary data assessments, derived from locals scale investigations of mountain environments, lead to the conclusion that a more inclusive valuation of the forest ecosystems in the municipality may exceed the current total economic value by at least $80 \%$.

In conclusion, serious prerequisites exist within the limits of the Municipality of Chepelare to economically "absorb" the very high ecological assets, as well as the specificities and multi-functionality of its forest ecosystems and grazing lands, particularly for development of alternative types of tourism and local production of clean, renewable, and eco- products. Special attention should be paid to targeted monitoring and valuation of the regulating services, particularly ecosystem functions related to water purification, which will affect the market price of water and forest 
resources, as well as the services related to generic resources, like medicinal plants, game species, mushrooms, honey, and berries.

\section{Acknowledgements}

This research is sponsored by the "National, European, and Civilizational Dimensions of the Culture - Language - Media Dialogue" Program of the "Alma Mater" University Complex in the Humanities at Sofia University "Saint Kliment Ohridski", funded by the Bulgarian Ministry of Education and Science - Bulgarian Science Fund.

\section{References}

Barbier, E.B. 2008. Ecosystems as Natural Assets. Foundations and Trends in Microeconomics 4(8):611-681

Brouwer et al. (2013). R. Brouwer, L. Brander, O. Kuik, E. Papyrakis, I. Bateman. A synthesis of approaches to assess and value ecosystem services in the EU in the context of TEEB. TEEB follow-up study for Europe.

Burkhard et al., (2014). Burkhard B, M.Kandziora, Y.Hou, F. Müller. Ecosystem Service Potentials, Flows and Demands - Concepts for Spatial Localisation, Indication and Quantification LANDSCAPE ONLINE 34:1-32 (2014), http://dx.doi.org/10.3097/LO.201434

CICES v4.3, MAES (2013). Mapping and Assessment of Ecosystems and their Services. An analytical framework for ecosystem assessments under Action 5 of the EU Biodiversity Strategy to 2020. Discussion paper - Final, April 2013. European Commission. http://dx.doi.org:10.2779/12398

CIS for WFD (2004). Common Implementation Strategy for the Water Framework Directive 2000/60/EC (2004): Groundwater body characterization - Technical report on groundwater body characterization issues, April 2004

EABD 2010-2015, Management plan for the river basins in the East Aegean Basin Directorate 2010-2015, Ministry of Environment and Waters, Bulgaria

Executive Environment Agency, Ministry of Environment and Water http://eea.government.bg/bg/projects/korine-14/kzp-danni-clc-data

Forman, R.T.T., (1995). Land Mosaics: The Ecology of Landscapes and Regions, Cambridge Univ. Press, Cambridge.

Hayha T. (2014) Mapping ecosystem services: an integrated biophysical and economic evaluation. YSSP Interim Report IR-14-007, International Institute for Applied Systems Analysis, Austria

Honey-Rosés J., L.H. Pendleton (2013). A demand driven research agenda for ecosystem services. Ecosystem Services 5:160-162

Kozhuharov D., E. Kozhuharova, R. Marinova, N. Katscov, J. Yanev (1991). Geological map of Bulgaria, scale 1:50 000, Chepelare

Kozhuharov D., R. Marinova, N. Katscov (1989). Geological map of Bulgaria, scale 1:50 000, Smolyan

MDP 2014-2020, Municipal Development Plan of the Chepelare Municipality 2014-2020, BG051PO002/ 13/1.3-07, 2015, Chepelare

Muller F., B. Burkhard (2012). The indicator side of ecosystem services. Ecosystem Services 1:26-30

NSI (2012). National Statistical Institute, Republic of Bulgaria: Regional statistics (data series) http://www.nsi.bg/en/content/4247/prices-agricultural-production-data-series

NSI (2015a). National Statistical Institute, Republic of Bulgaria: Prices of agricultural production 2015 (data series) http://www.mzh.government.bg/MZH/en/ShortLinks/SelskaPolitika/Agrostatistics.aspx

NSI (2015b), National Statistical Institute - National Statistical Book-2015, Sofia

Price list of Hvoyna State Foresty, 2014, Chepelare

TEEB (2010). The Economics of Ecosystems and Biodiversity: The Ecological and Economic Foundations http://www.teebweb.org/publication/the-economics-of-ecosystems-and-biodiversity-teebecological-and-economic-foundations/ 